\title{
Persistent homology analysis of craze formation
}

\author{
Takashi Ichinomiya \\ Gifu University School of Medicine, Yanagido 1-1, Gifu, Gifu 501-1194, Japan \\ and United Graduate School of Drug Discovery and Medical Information Sciences, Gifu University, Yanagido 1-1, Gifu, Gifu 501-1194, Japan
}

Ippei Obayashi and Yasuaki Hiraoka

Tohoku University Advanced Institute for Materials Research, 2-1-1 Katahira, Aoba-ku, Sendai, 980-8577 Japan

(Received 17 November 2016; revised manuscript received 25 December 2016; published 13 January 2017)

\begin{abstract}
We apply a persistent homology analysis to investigate the behavior of nanovoids during the crazing process of glassy polymers. We carry out a coarse-grained molecular dynamics simulation of the uniaxial deformation of an amorphous polymer and analyze the results with persistent homology. Persistent homology reveals the void coalescence during craze formation, and the results suggest that the yielding process is regarded as the percolation of nanovoids created by deformation.
\end{abstract}

DOI: 10.1103/PhysRevE.95.012504

\section{INTRODUCTION}

Understanding the microscopic process of yielding is a fundamental problem in material science. The yielding of a glassy polymer occurs either through shear deformation or through crazing [1]. Crazing is a unique phenomenon observed in glassy polymers, and the process of craze formation has been intensively investigated by electron microscopy [2], optical microscopy [3], atomic force microscopy [4], and many other methods. On the basis of these observations, several kinetic models of craze formation have been proposed [5-7].

Recent advances in molecular dynamics (MD) simulations have provided new methods for studying the atomic-scale process of craze formation. Although a full-atomic MD simulation is currently impossible due to the large simulation size required to reproduce craze formation, coarse-grained MD (CG-MD) simulations have successfully reproduced craze formation processes [8-11]. CG-MD simulations enable the investigation of the atomic-scale dynamics of craze formation, which is difficult to detect by experiments.

Although CG-MD simulations have made important contributions to the understanding of craze formation, the relation between the kinetic models and the CG-MD simulations still remains unclear. This is partially due to the lack of the definition of voids in the CG-MD simulations. In kinetic models of craze initiation, the material is often assumed to be a continuum, and the voids play an essential role. On the other hand, CG-MD simulations represent materials as a set of beads. From this viewpoint, the material is not a continuum, and it is difficult to define the voids. To gain information about the voids from an MD simulation, several ideas have been proposed such as the nonaffine displacement or Voronoi volume $[12,13]$. However, variables are assigned to each bead in these methods, and they do not provide direct information about the voids, such as the positions, sizes, or shapes.

Published by the American Physical Society under the terms of the Creative Commons Attribution 4.0 International license. Further distribution of this work must maintain attribution to the author(s) and the published article's title, journal citation, and DOI.
To overcome this difficulty, we employ a persistent homology $(\mathrm{PH})$ analysis in this study. $\mathrm{PH}$ is an emerging method for investigating the hidden structure from complex data $[14,15]$. This method shows good performance even when the system has no clear structure and has provided successful results for the analysis of glass or proteins [16-18]. A PH analysis has several desirable properties for investigating the behavior of voids. First, the variables obtained from the $\mathrm{PH}$ analysis are strongly related to the size of the voids. In particular, the variable called "deaths" can be regarded as the size of the voids. Second, these variables are defined for the set of beads. This implies that, when we choose a void, we can obtain the beads that form the void. Therefore the sizes, positions, and shapes of the voids are obtainable by $\mathrm{PH}$.

In this study, we carry out a CG-MD simulation of the uniaxial deformation of a glassy polymer and investigate the sizes, shapes, and number of nanovoids using PH. The results show that the number of nanovoids increases as the strain increases and rapidly decreases after yielding. An investigation of the large voids emerging after yielding reveals that these nanovoids have a complex structure and are created by the coalescence of smaller voids. These results suggest that yielding is regarded as the "percolation of nanovoids".

This paper is organized as follows. In Sec. II, we describe the simulation model and $\mathrm{PH}$ analysis. The results are presented in Sec. III. Finally, in Sec. IV, we summarize our results, discuss the problems to be solved, and present the possible applications of $\mathrm{PH}$.

\section{METHOD}

In this section, we describe the details of the CG-MD simulations and $\mathrm{PH}$ analysis.

\section{A. Simulation of craze formation}

On the basis of the previous work by Rottler and Robbins [9], we employed the Kremer-Grest model as the coarsegrained model of glassy polymers. In our model, the system consists of 512 polymer chains, and each chain contains 512 beads of mass $m$. The bonding and bond-bending potentials 


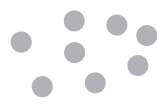

(a)

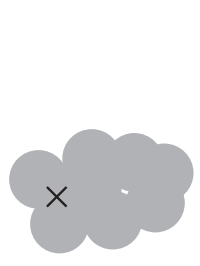

(d)

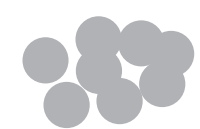

(b)

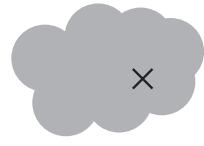

(e)

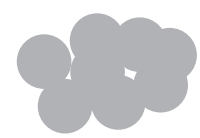

(c)

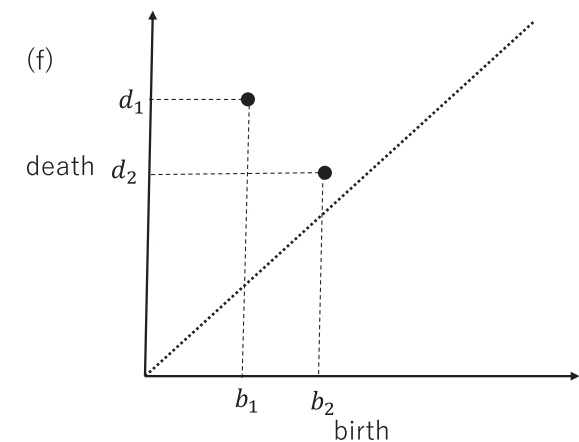

FIG. 1. Schematic representation of persistent homology. (a) When the radius of each bead $r$ satisfies $r^{2}<b_{1}$, all beads are disconnected. At $r^{2}=b_{1}$ shown in (b), a loop emerges. In (c), another loop emerges at $r^{2}=b_{2}$. The latter loop disappears at $r^{2}=b_{2}$ in (d) by meeting balls at the "death position" denoted by the cross mark. In a similar way, the former disappears at $r^{2}=d_{1}$ in (e). This persistent homology is represented by the persistent diagram shown in (f).

$V_{\text {bond }}$ and $V_{\text {bend }}$ are given by

$$
V_{\text {bond }}=u_{b}\left(r-R_{1}\right)^{3}\left(r-R_{0}\right)
$$

and

$$
V_{\text {bend }}=-\frac{u_{a}}{2}(\cos \theta-1)^{2},
$$

where $u_{b}, R_{1}, R_{0}$, and $u_{a}$ are parameters that define the potential functions, and $r$ and $\theta$ represent the distance between beads and the angle between two connected bonds, respectively. The potential between an unbonded pair of atoms is given by the Lennard-Jones potential,

$$
V_{\mathrm{LJ}}=4 u_{L}\left[\left(\frac{a}{r}\right)^{12}-\left(\frac{a}{r}\right)^{6}\right]
$$

In the simulation, we normalize the parameters as $m=1.0, u_{L}=1.0$, and $a=1.0$. We set $R_{0}=1.5, R_{1}=$ $0.7575, u_{a}=1.0$, and $u_{b}=2351$. In this model, $V_{\text {bond }}$ takes a minimum at $r=0.96$. We note that in real materials, such as polystyrene or polymethylmethacrylate, $a \sim 0.8-1.5 \mathrm{~nm}$.

Using these potentials, we carried out a simulation of uniaxial deformation. First, we set the pressure $P=1.0$ and the temperature $T=1.0$ and carried out a simulation under a constant pressure and temperature to create melted polymers. After relaxing the system to the equilibrium state, we gradually cooled the system from $T=1.0$ to $T=0.3$ to obtain the glassy polymer. We plot the relation between $T$ and the volume to assert that the system is not liquid but glassy. After cooling, we carried out uniaxial deformation at a constant rate $d L_{z} / d t=0.1 a$ with the Poisson ratio $R=0$.

In the simulations of deformation, we employed periodic boundary conditions. The simulations are carried out by COGNAC 8.3 in the Open Computational Tool for Advanced Material Technology (OCTA) [19].

\section{B. Persistent homology analysis of the set of beads}

In this subsection, we describe the $\mathrm{PH}$ analysis employed in this study. The general mathematical definition of $\mathrm{PH}$ has been presented in a textbook [15], and we only describe the $\mathrm{PH}$ of the $\alpha$ complex that we employ for the analysis of the CG-MD results.

Suppose that there are $k$ beads at $x_{1}, x_{2}, \ldots, x_{k}$, as shown in Fig. 1(a). In the PH analysis, we represent each bead by a ball with a radius $r$. If $r$ is small, then all spheres are disconnected, as shown in Fig. 1(a). As $r$ increases, the spheres coalesce to create a "chain", and a loop-a chain of the beads that surround empty space-emerges at $r^{2}=b_{1}$ [Fig. 1(b)]. A further increase in $r^{2}$ creates one more loop [Fig. 1(c)]. However, the empty spaces surrounded by these loops are covered by spheres if $r^{2}$ is sufficiently large. At $r^{2}=d_{2}$, the balls meet at the "death position" denoted by the cross mark in Fig. 1(d), and the second loop disappears. The first loop also disappears at $r^{2}=d_{1}$, as shown in Fig. 1(e). This example shows that every loop $i$ has a "birth" and "death", $b_{i}$ and $d_{i}$, and the loop exists only when $b_{i}<r^{2}<d_{i}$. We also note that the death position is uniquely defined for each loop.

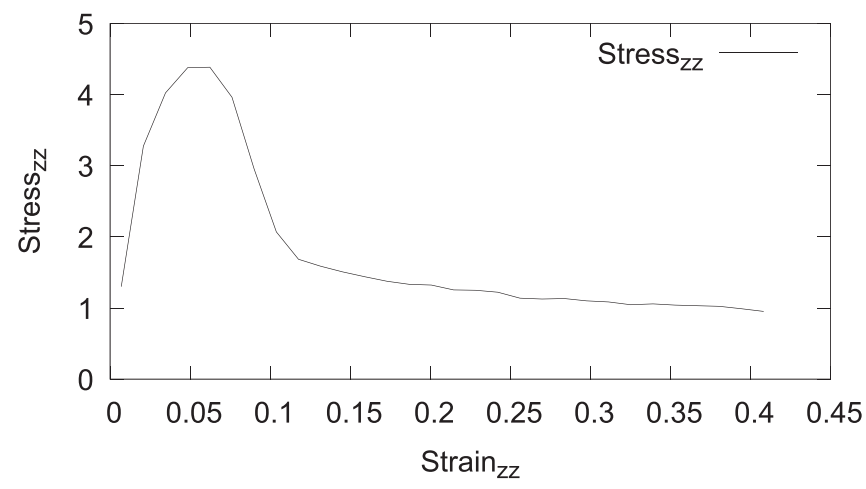

FIG. 2. Stress-strain curve obtained by CG-MD simulation. 


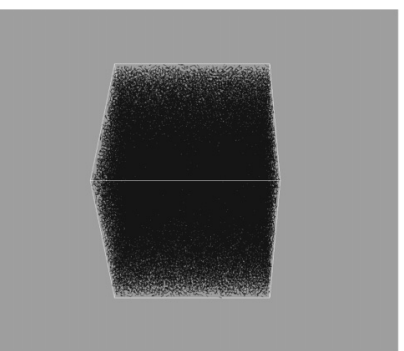

(a)

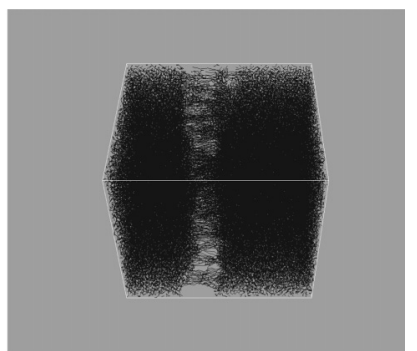

(b)
FIG. 3. Snapshot of the MD simulation (a) before and (b) after yielding. (a) $\epsilon=0.007$ and (b) $\epsilon=0.20$ ).

$\mathrm{PH}$ is the map from $\left\{x_{1}, x_{2}, \ldots, x_{k}\right\}$ to $\left.\left\{\left(b_{1}, d_{1}\right),\left(b_{2}, d_{2}\right), \ldots,\left(b_{l}, d_{l}\right)\right)\right\}$, where $l, b_{j}$, and $d_{j}$ are the number of loops and the "birth" and "death" of the $j$ th loop, respectively. In this example, we represent the PH of the holes, whose boundaries are given by one-dimensional chains. Therefore, we call it the $D=1 \mathrm{PH}$ where $D$ represents the dimension of the boundaries. In the following analysis, we employ the $D=2 \mathrm{PH}$ that concerns the set of beads that surround voids, which can be defined in the same manner. To represent the results of the $\mathrm{PH}$ analysis, we employ the scatter plot of $\left(b_{i}, d_{i}\right)$ shown in Fig. 1(f). This plot is called a "persistent diagram".

From the definition, it is clear that the $D=2 \mathrm{PH}$ analysis gives information about the voids. If there is a large spherical void with a radius $R$, then it implies that there is a point in the persistent diagram whose death is about $R^{2}$. Moreover, we can inspect the form of the voids using the $\mathrm{PH}$ analysis. In the PH, "births" and "deaths" are defined for "hole boundaries", which are constructed from the set of beads. Although the beads comprising a boundary cannot be uniquely determined, a recently developed numerical method allows the "optimal cycle", the minimum set of beads for constructing each boundary, to be obtained [20]. Hence, PH is suitable for quantitative and qualitative analyses of the voids in glassy polymers.

Before concluding this section, we note several limitations of the PH analysis. First, the size of the voids estimated by $\mathrm{PH}$ is semiquantitative. Voids are three-dimensional structures

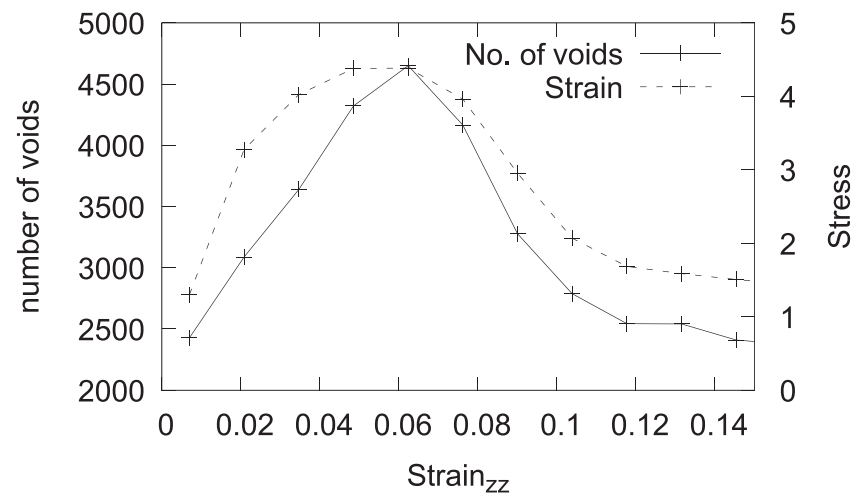

FIG. 5. Plot of the number of voids versus the strain. The dashed line represents the stress-strain curve.

and may have a strong anisotropy. In this case, the definition of the "size" of a void has a large ambiguity. Therefore, death should not be regarded as a flawless quantitative measure of the size of a void. Second, there are many points in the persistent diagram that have no relation with physical voids. The points near the diagonal line, $b_{i}-d_{i}$, are supposed to have no physical meanings because these points disappear due to small thermal fluctuations. Other points that have no relation with the voids are those with a large birth $b_{i}$. In the case of a glassy polymer, it is natural to assume that voids are surrounded by beads, whose distance is on the order of the bonding length. Therefore, the points with a large $b_{i}$ will not correspond to physical voids, even if they have some physical meaning.

\section{RESULTS}

\section{A. Simulation of craze formation}

Before presenting the results of the $\mathrm{PH}$ analysis, we investigate the results of a CG-MD simulation to confirm that our model reproduces the yielding and crazing processes. Figure 2 shows the stress-strain curve obtained by our simulation. The stress $\sigma$ increases as the strain $\epsilon$ increases for a small $\epsilon$ and has a sharp peak at $\epsilon \sim 0.05$. This figure suggests that yielding occurs at $\epsilon \sim 0.05$. To confirm that crazing emerges after yielding, we show the configuration of the beads before

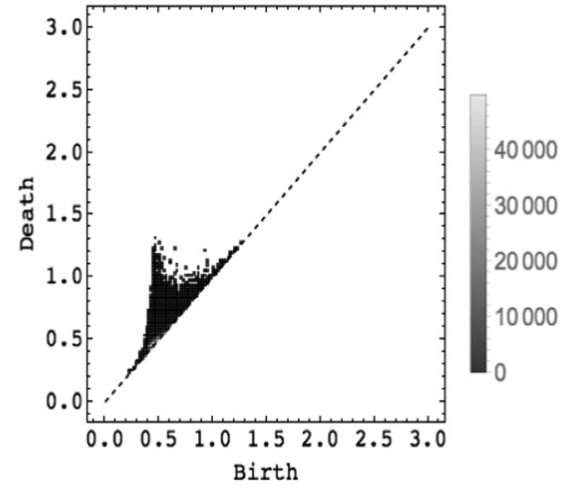

(a)

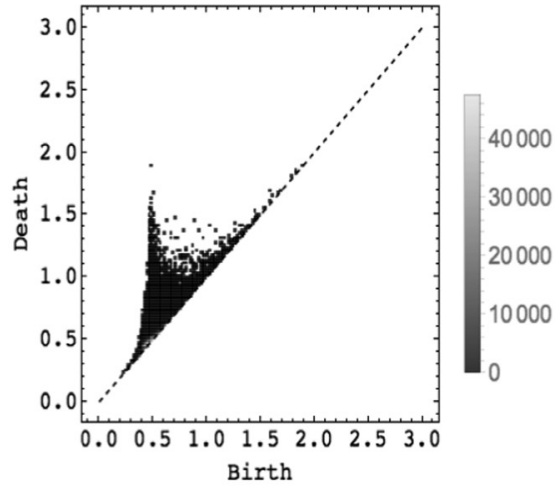

(b)

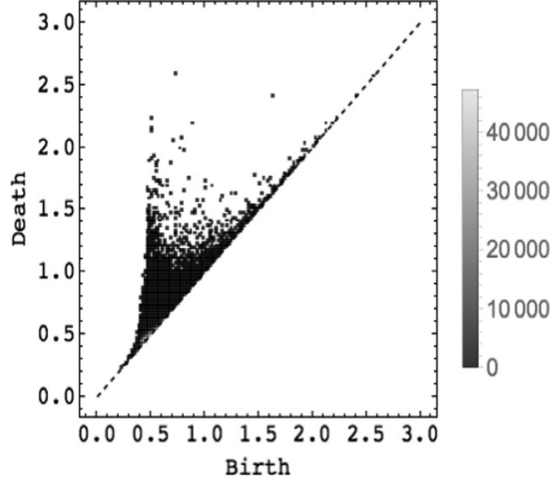

(c)

FIG. 4. $D=2$ persistent diagram obtained from simulation: (a) and (b) before and (c) after yielding. Dashed lines represent diagonal lines. (a) $\epsilon=0.007$, (b) $\epsilon=0.049$, and (c) $\epsilon=0.062$ 


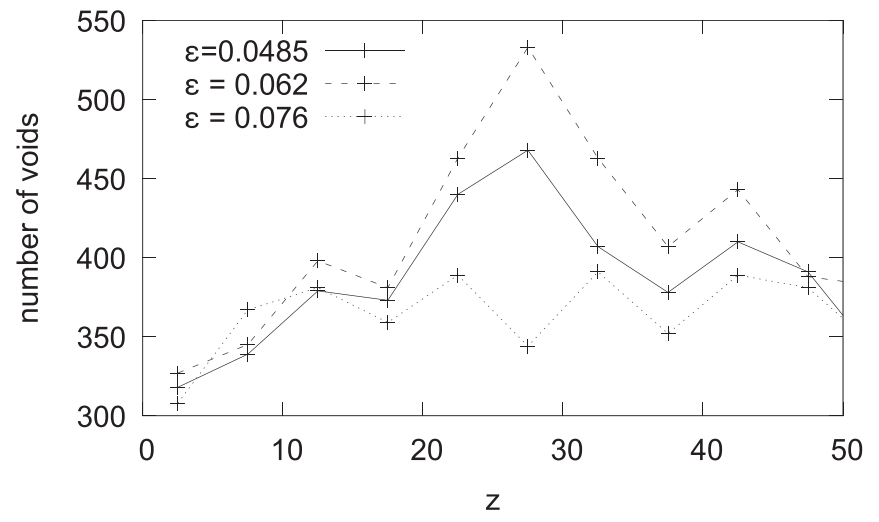

FIG. 6. Distribution of the voids along the $z$ axis at $\epsilon=$ $0.0485,0.062$, and 0.076 .

and after yielding in Fig. 3. In the case of $\epsilon=0.007$ before yielding, we observe no clear structure, whereas in the case of $\epsilon=0.20$ after yielding, we observe a large empty space and fibrils that connect both sides of the empty space. These results are similar to the structure of the crazes observed in various experiments. Therefore, we conclude that our model reproduces the formation of a craze successfully.

\section{B. Persistent homology analysis}

In Fig. 4, we present density plots of the $D=2$ persistent diagrams at $\epsilon=0.007,0.049$, and 0.062. As noted in Sec. II, the points close to the diagonal line and those with large births cannot be interpreted as voids. Because we set the natural bond length as 0.96 , it would be natural to assume that the births of voids are $O(1)$. In the following, we carried out an analysis assuming that the births of voids are less than 0.8 . We have confirmed that the results do not change qualitatively if the cutoff of births is 1.0 or 0.6 .

When $\epsilon$ is less than yielding point, we find that the points with a large death gradually increase as $\epsilon$ increases. However, after yielding, the points with a large death quickly increase. For example, at $\epsilon=0.049$, i.e., before yielding, we find no point that satisfies both $b<0.8$ and $d>2.0$, whereas at $\epsilon=$ 0.0623 , there are six points that satisfy these conditions after yielding.

The changes in the persistent diagram before and after yielding are more clear when we count the number of voids. In Fig. 5 we plot the number of points that satisfy $b<0.8<d$ along with the stress-strain curve. Clearly, the number of voids shows a peak at $\epsilon=0.062$, which is close to the yielding point $\epsilon \sim 0.05$. This figure suggests that a clear change in the microstructure occurs at the yielding point.

To understand the reason for the emergence of the peak at yielding, we investigate the "position" of voids before and after yielding. Because voids have a complex structure in general, there exists ambiguity in the definition of the "position" of the voids. In this analysis, we use the "death position" as the position of the voids. The death position is defined as the position at which the balls meet when a void dies, denoted by the cross in Fig. 1. There are several advantages to the use of this position as the position of the voids. First, the "death position" is always inside of the voids. Therefore, if the voids are small, it is natural to use this point as the position of the voids. Second, the computational cost for calculating the death position is much smaller than that of the optimal cycle calculation. When we calculate the time of death of a void, we always identify four balls that met at the time of death. Therefore, the death position is easily calculated because it is the centroid of these four beads.

In Fig. 6, we show the distribution of the voids along the $z$ axis at $\epsilon=0.0485,0.062$, and 0.076 . This figure shows that the change in the number of voids is localized at $z=25-30$. At $\epsilon=0.0485$ before yielding, the number of voids has a dull peak at $z=25-30$. As $\epsilon$ increases, this peak becomes sharp and suddenly decreases at $\epsilon=0.076$. This figure shows that a structural change occurs in this region.

The structural change at $z=25-30$ becomes more clear by an optimal cycle calculation to obtain the form of the voids. Because the optimal cycle calculation has a high computational cost, we only investigate the large voids that satisfy $b<0.8$ and $d>2.0$ at $\epsilon=0.062$. The births and deaths are listed in Table I.

The location of the beads that construct these optimal cycles are plotted in the $x z$ plane in Fig. 7. All beads are placed around $z=25-30$, i.e., the region where the number of voids changes by yielding. Therefore, the structural change observed in the number of voids is the emergence of large voids.

The shapes of these voids are colored in light gray in Fig. 8. The number of beads needed for the creation of each optimal cycle is listed in Table I. The shapes of these voids are complex and highly anisotropic. These complex forms of the voids imply that it is difficult to estimate the "size" of the voids. For example, the sixth cluster, which is composed of 3034 beads, has a smaller death than the third cluster, which is composed of 192 beads. This implies that the deaths should not be regarded as a flawless measure of the size of the voids.

To investigate how these large voids emerge, we carry out a $\mathrm{PH}$ analysis of the beads engaged in each void before yielding.

TABLE I. Characteristics of voids that satisfy both $b<0.8$ and $d>2.0$ at $\epsilon=0.062$ : births, deaths, the number of surrounding beads, the number of voids before yielding given by the beads engaged in each void, and the average Voronoi volume.

\begin{tabular}{lccccc}
\hline \hline ID & Birth & Death & Number of beads that surround voids & Number of voids before yielding & Average Voronoi volume of beads \\
\hline 1 & 0.712 & 2.04 & 143 & 11 & 1.068 \\
2 & 0.502 & 2.12 & 307 & 5 & 0.788 \\
3 & 0.734 & 2.59 & 192 & 5 & 1.059 \\
4 & 0.789 & 2.07 & 2246 & 4 & 1.070 \\
5 & 0.509 & 2.22 & 3034 & 63 & 0.746 \\
6 & 0.517 & 2.16 & 97 & 0.748 \\
\hline \hline
\end{tabular}




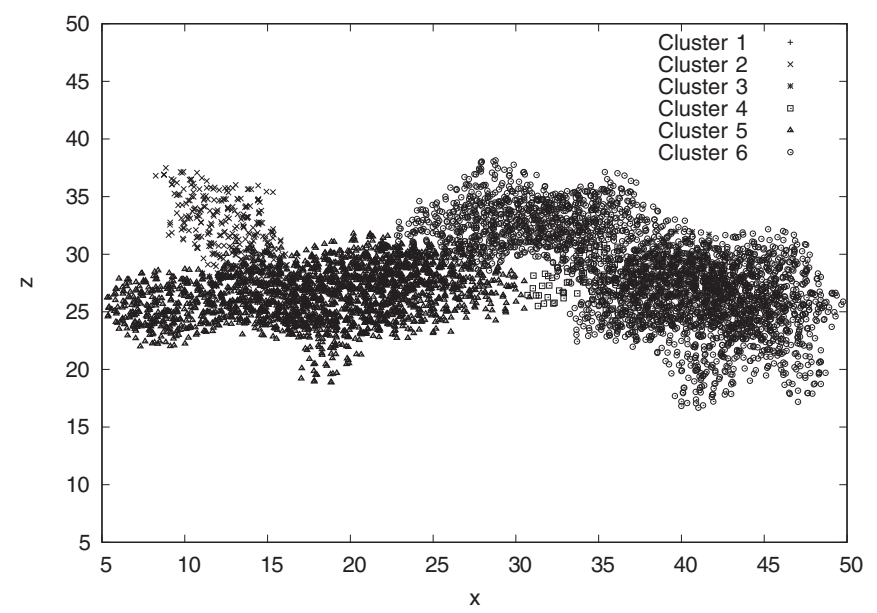

FIG. 7. Plot of the positions that construct large voids listed in Table I in the $x z$ plane.

First, we identify the beads inside or on the surface of each void. Then, we obtain the positions of these beads at $\epsilon=0.049$ before yielding and carry out a PH analysis of these beads to obtain a list of voids. The result is indicated in dark gray in Fig. 8. This figure shows that these beads construct several voids before yielding. The number of voids before yielding is listed in Table I. The results suggest that large voids emerge through the coalescence of many smaller voids.

The results in Fig. 5 and 8 suggest that the decrease in the number of voids after yielding is caused by the coalescence of small voids. When the amount of deformation is small, the number of small voids increases as the strain increases. For this small amount of deformation, void coalescence may occur, but it will not be very frequent, such that the total number of voids increases. However, these small voids coalesce at yielding to create large voids, which cause a sharp decrease in the number of small voids. This yielding scenario reminds us of a percolation transition. Our results suggest that yielding and craze formation can be regarded as the emergence of a large void by the percolation of small voids.

Finally, we compare our result with a conventional Voronoi tessellation analysis. Because a plastic polymer glass is a

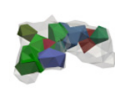

(a)

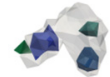

(d)

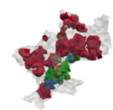

(e)

FIG. 8. The forms of large voids listed in Table I before and after yielding. Light-gray transparent polygons represent large voids after yielding, and opaque dark-gray polygons (color online) represent voids before yielding. The different colors indicate different voids. (a) Cluster 1, (b) cluster 2, (c) cluster 3, (d) cluster 4, (e) cluster 5, and (f) cluster 6 .

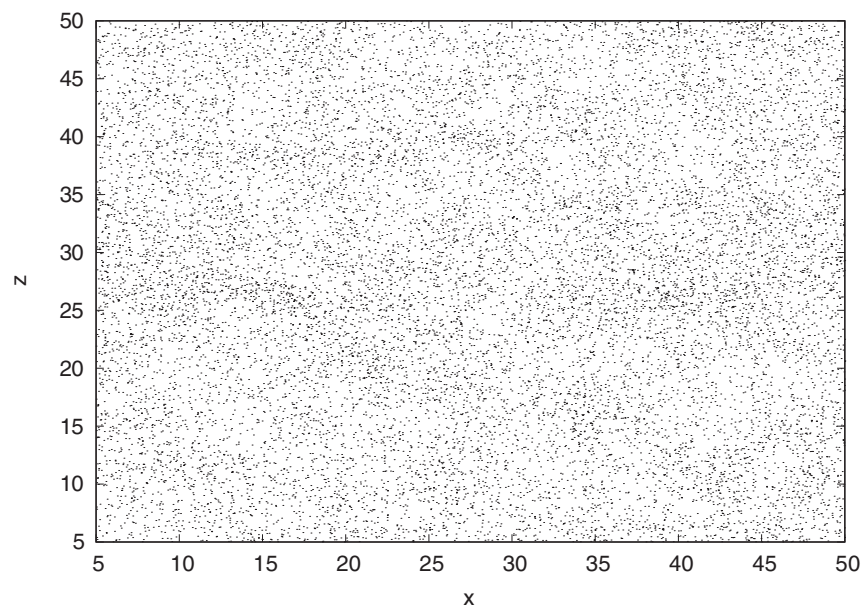

FIG. 9. Plot of positions of beads that have a Voronoi volume larger than 1.0 in the $x z$ plane.

strongly disordered material, it is difficult to classify the shapes of the Voronoi polyhedrons. In previous studies of crazing, the Voronoi volume has been used for the investigation of crazing $[12,13]$. We calculate the Voronoi volumes of all beads at $\epsilon=0.062$ after yielding. In this case, the average Voronoi volume is 0.649 . In Table I, we show the average Voronoi volume of the beads that are engaged in each void. The average volume is between 0.748 and 1.070, which is higher than the average Voronoi volume. However, we also note that a large Voronoi volume does not always imply craze formation. For example, we plot the position of beads that have Voronoi volumes larger than 1.0 in the $x z$ plane in Fig. 9. The beads with large Voronoi volume are widely spread, and it is difficult to identify the crazes in this plot. This figure implies that we need a more complicated method to recognize craze formation using a Voronoi tessellation. On the other hand, Fig. 7 clearly indicates the location of a craze. Therefore, $\mathrm{PH}$ gives a simple and straightforward method to investigate craze formation.

\section{DISCUSSION AND CONCLUSION}

In this study, we applied a PH analysis to the CG-MD simulation of craze formation. We revealed that the number of voids shows a clear peak at the yielding point. An investigation of large-void formation implies that a decrease in the number of voids is caused by the coalescence of small voids.

Void coalescence is widely accepted as essential to craze formation in polymer glasses [21,22]. However, this process has not been reproduced by MD simulations. To the best of the authors' knowledge, our work is the first MD simulation that confirms void coalescence. Moreover, our results suggest the similarity between yielding and percolation, which has not been reported until now.

Our result implies that the coalescence process of nanovoids should be included in the kinetic theory of craze formation. In the most successful kinetic theory by Argon and Hannoosh [5], the growth of a craze is driven by the meniscus instability. In this scenario, the growth of voids is not caused by coalescence but by the strong stress at the edges of the voids. 
Although this scenario successfully explains the structure of crazes at a scale of $O(10 \mathrm{~nm})$, our result indicates that the scenario should be modified at a smaller scale. The coalescence of nanovoids has been theoretically studied by several researchers [23,24], but most of these works assert that the nanovoids are periodically placed. To construct a more realistic model of craze formation, the theory of the coalescence of randomly distributed nanovoids will be needed.

To confirm the yielding scenario proposed in this paper, an experiment with real material is indispensable. Our results suggest that there is a change in the free-volume distribution at yielding. Positron annihilation lifetime spectroscopy (PALS) is a powerful method for obtaining information related to the free volume [25]. Although it is difficult to obtain the size of strongly anisotropic cavities, as shown in Fig. 8(f), a PALS study of craze formation will be useful to validate our suggestions.

Further study with $\mathrm{PH}$ will provide more valuable insights into the yielding process. For example, a better definition of the size of the voids would be obtained. As we have shown, $\mathrm{PH}$ has some flaws when defining the size of the voids using deaths. The results in Table I suggest that the number of beads in the optimal cycle may be a better measure of the size of voids. Another interesting question is related to the spatial distribution of the voids. In this paper, we showed that the number of voids has a dull peak at the position where crazing occurs before yielding. A more detailed study of the spatial distribution of the nanovoids will make significant contribution toward estimating the strength of materials. Unfortunately, it is difficult to carry out this analysis because a large computational power is necessary to calculate the optimal cycles. We will need further development of $\mathrm{PH}$ algorithms.

The PH analysis would be useful for the analysis of other materials and phenomena. Void coalescence has been regarded as essential for the ductile fracture of metals [26]. PH will give essential insights into this process. With regard to polymer science, there will be at least two applications. First, the fracture process of thermosetting polymers will be interesting. The tensile behaviors of thermosetting polymers have been investigated by CG-MD simulation [27]. A PH analysis will give insights into the yielding process of these materials. Another important target of the PH analysis is shear yielding. In shear yielding, the volume of the system is conserved, in contrast to crazing. A trial for capturing the structural changes in shear yielding by $\mathrm{PH}$ is a challenging problem.

\section{ACKNOWLEDGMENTS}

We acknowledge Emerson Escolar for his help with the calculation of the optimal cycles. We also acknowledge Takashi Kuriyama, Shin-etsu Fujimoto, and Yasumasa Nishiura for their valuable comments. This work was financially supported by the Cross-ministerial Strategic Innovation Promotion Program promoted by the Cabinet Office, Government of Japan, and the Japan Science and Technology Agency, and CREST by Japan Science and Technology Agency.
[1] A. J. Kinloch and R. J. Young, Fracture Behaviour in Polymers (Elsevier, London, 1983).

[2] R. P. Kambour, MacroMol. Rev. 7, 1 (1973).

[3] S. S. Sternstein, in Treatise on Materials Science and Technology, edited by J. M. Schultz, (Academic Press, New York, 1977) Vol. 10, p. 541.

[4] C. Thomas, V. Ferreiro, G. Coulon, and R. Seguela, Polymer 48, 6041 (2007).

[5] A. S. Argon and J. Hannoosh, Philos. Mag. 36, 1195 (1977).

[6] A. S. Argon, Polymer 52, 2319 (2011).

[7] C. B. Bucknall, Polymer 48, 1030 (2007).

[8] J. Rottler, S. Barsky, and M. O. Robbins, Phys. Rev. Lett. 89, 148304 (2002).

[9] J. Rottler and M. O. Robbins, Phys. Rev. E 68, 011801 (2003).

[10] D. K. Mahajan and A. Hartmaier, Phys. Rev. E 86, 021802 (2012).

[11] S. Venkatesan and S. Basu, J. Mech. Phys. Solids 77, 123 (2015).

[12] B. Sixou, Mol. Simul. 33, 965 (2007).

[13] A. Makke, M. Perez, J. Rottler, O. Lame, and J.-L. Barrat, Macromol. Theory Simul. 20, 826 (2011).

[14] H. Edelsbrunner, D. Letscher, and A. Zomorodian, Discrete Comput. Geom. 28, 511 (2002).
[15] H. Edelsbrunner and J. Harar, Computational Topology: An Introduction (American Mathematical Society, Providence, RI, 2010).

[16] Y. Hiraoka, T. Nakamura, A. Hirata, E. G. Escolar, K. Matsue, and Y. Nishiura, Proc. Natl. Acad. Sci. USA 113, 7035 (2016).

[17] M. Gamerio, Y. Hiraoka, S. Izumi, M. Kramar, K. Mischaikow, and V. Nanda, Jpn. J. Indust. Appl. Math. 32, 1 (2015).

[18] K. Xia and G. W. Wei, Int. J. Num. Methods Biomed. Eng. 30, 814 (2014).

[19] Computer Simulation of Polymeric Materials, edited by the Japan Association for Chemical Innovation (Springer, Singapore, 2016).

[20] E. G. Escolar and Y. Hiraoka, in Optimization in the Real World: Toward Solving Real-World Optimization Problems, edited by K. Fujisawa, Y. Shinano, and H. Waki (Springer, Tokyo, 2016).

[21] L. Cheng and T. F. Guo, Int. J. Solids Struct. 44, 1787 (2007).

[22] A. C. Steenbrink, E. Van Der Glessen, and P. D. Wu, J. Mech. Phys. Sol. 45, 405 (1997).

[23] A. Needleman, J. Appl. Mech. 39, 964 (1972).

[24] A. L. Gurson, J. Eng. Mater. Technol. 99, 2 (1977).

[25] R. B. Gregory, J. Appl. Phys. 70, 4665 (1991).

[26] A. Amine Benzerga and J.-B. Leblond, Adv. Appl. Mech. 44, 169 (2010).

[27] S. Yang and J. Qu, Phys. Rev. E 90, 012601 (2014). 